\title{
Efficacy of COVID-19 vaccination on the symptoms of patients with long COVID: a target trial emulation using data from the ComPaRe e-cohort in France
}

Viet-Thi Tran ( $\boldsymbol{D}$ thi.tran-viet@aphp.fr)

Université de Paris https://orcid.org/0000-0003-1863-6739

Elodie Perrodeau

Assistance Publique Hôpitaux de Paris

Julia Saldanha

Université de Paris

Isabelle Pane

Assistance Publique Hôpitaux de Paris

Philippe Ravaud

Paris Descartes University

\section{Article}

Keywords: COVID-19, long COVID, COVID-19 vaccination, comparative effectiveness research, causal inference, target trial emulation

Posted Date: February 17th, 2022

DOI: https://doi.org/10.21203/rs.3.rs-1350429/v1

License: (c) (1) This work is licensed under a Creative Commons Attribution 4.0 International License. Read Full License 


\section{Abstract}

We used data from 910 adult patients included the ComPaRe long COVID cohort to emulate a target trial evaluating the effect of vaccination on the symptoms of patients who already had long COVID. We used propensity score matching to compare the outcomes, at 120 days, of patients who received a first COVID19 vaccine injection $(n=455)$ and those who did not $(n=455)$. Vaccination reduced the number of long COVID symptoms (mean difference: $-1.8,95 \% \mathrm{Cl}-3.0$ to -0.5 ), the proportion of patients with an unacceptable symptom state (risk difference $-7.5 \%, 95 \% \mathrm{Cl}-14.4$ to -0.5 ), and doubled the remission rate (16.6\% vs $7.5 \%$, HR: $1.97,95 \% \mathrm{Cl} 1.23$ to 3.15$)$. In the vaccination group, two (0.4\%) patients reported serious adverse events leading to hospitalisation. Vaccination lowered the severity and life impact of long COVID, at 120 days, among patients with persistent symptoms.

\section{Background}

As of January 2022, about 355 million people worldwide have been infected by the severe acute respiratory syndrome coronavirus 2 (SARS-CoV-2), the pathogen responsible for the coronavirus disease 2019 (Covid-19). Most Covid-19 cases are acute and resolve within 14 days. However, according to the United Kingdom Office for National Statistics, about 10\% of patients will experience "long COVID" or "post COVID-19 disease", that is, the persistence of symptoms for several months after their initial symptoms ${ }^{1}$ 2 .

Long COVID can involve multiorgan dysfunction and have a severe impact on patients' lives. $^{3}$ One year after onset, more than $85 \%$ of patients with long COVID still report symptoms, and $67 \%$ have not returned to previous levels of work 45 .

To date, management of patients with long COVID relies solely on general advice, supported selfmanagement, and multidisciplinary rehabilitation ${ }^{6}$. No specific treatment has been identified. Some researchers recently suggested that COVID-19 vaccination might improve patients' symptoms by eliminating potential viral reservoirs and/or viral fragments. ${ }^{7}$ Only sparse evidence currently supports this hypothesis. A non-peer reviewed of 900 patients with long COVID in the UK reported that $56.7 \%$ of participants perceived an improvement in their symptoms after the first COVID-19 vaccine injection ${ }^{8}$. A small-scale study found a modest overall improvement in the symptoms of 44 patients with long COVID vaccinated against COVID-19, compared with 22 unvaccinated patients ${ }^{9}$. Finally, a recent cross sectional study found that vaccinated patients reported, on average, 50\% less post-acute COVID-19 symptoms than unvaccinated patients ${ }^{10}$. Unfortunately, in this study, the vaccination status at the time of infection was unknown and therefore some individuals were infected prior to vaccination while others were vaccinated after.

In this study, we used data from a large ongoing prospective cohort of patients with long COVID to emulate a target trial evaluating the effect of vaccination on the symptoms and on the impact of the disease. 


\section{Results}

\section{Participants and treatment groups}

The ComPaRe long COVID cohort is an ongoing nationwide e-cohort of patients with long COVID in France, nested in the ComPaRe research program (www.compare.aphp.fr), an umbrella e-cohort of patients with chronic conditions ${ }^{11}$. In this study, we analysed the data from 910 adult patients $(\geq 18$ years old) with a confirmed or suspected COVID-19 infection; with symptoms persisting more than three months past the initial infection; and who reported at least one symptom attributable to long COVID at study baseline. Participants' median age was 47 years (interquartile range (IQR) 40 to 54); 733 (80.5\%) of participants were female; $545(60.1 \%)$ had a confirmed infection; and $81(8.9 \%)$ had been hospitalised during their acute COVID-19. The interval between symptom onset and study baseline was 10.7 months (IQR 6.4 to 12.4 ).

Among the 910 included patients, we classified 455 patients who received their first COVID-19 vaccine injection between baseline and 60 days in the "vaccination group". First vaccine injection involved the BNT162b2 mRNA vaccine $(n=359,78.9 \%)$; the ChAdOx1 vaccine $(n=48,10.5 \%)$; the mRNA-1273 vaccine $(n=47,10.3 \%)$, or the Ad26.COV2.S vaccine $(n=1,0.2 \%)$. The median interval between baseline and vaccination was 38 days (IQR 24 to 53). The 455 vaccinated patients were propensity score-matched to 455 patients who did not receive the vaccine in the same period and who were classified in the "control group" (Table 1, Supplementary material 1). Methods and details regarding the matching procedures are presented in Online Methods and in the Supplementary Material 2 to 5 . After matching, all variables included in the propensity score models had standardized differences below 10\% (Supplementary material 6).

\section{Follow-up and outcomes}

Patients were assessed at 60 and 120 days after their inclusion in the study (baseline). At each of these observation times, they received an online questionnaire asking if they still had symptoms related to COVID-19. Those reporting persisting symptoms completed the long COVID symptom tool (ST) and impact tool (IT), a pair of validated patient-reported instruments assessing respectively 53 long COVID symptoms and 6 dimensions of patients' lives that can be affected by the disease. ${ }^{12}$ Those reporting that they no longer had any symptoms are asked to specify the date when they first noticed the absence of symptoms.

During follow-up, 69 patients (7\%) were lost to follow-up (32 in the vaccination group and 37 in the control group). Furthermore, 275 (60.4\%) patients in the control group received vaccination during their follow-up and were censored at their vaccination date. The median interval between baseline and censoring was 90 days (IQR 72.5 to 105). 
At 120 days after baseline, $16.6 \%$ patients in the vaccination group $(n=57)$ reported a remission of all symptoms from long COVID, compared with $7.5 \%(n=27)$ in the control group (risk difference (RD): $9.1 \%$, 95\% confidence interval (Cl) 5.0 to 13.2 / hazard ratio (HR): 1.97, 95\% Cl 1.23 to 3.15, E-value 3.35) (Figure). Disease severity was significantly less severe in the vaccination group than in the control group. The long COVID ST score was 13.0 (9.4) in the vaccination group and 14.8 (9.8) in the control group (mean difference: $-1.8,95 \% \mathrm{Cl}-3.0$ to -0.5 ). The impact of long COVID on patients' lives was significantly lower in the vaccination group than in the control group. The mean (SD) long COVID IT score was 24.3 (16.7) in the vaccination group and 27.6 (16.7) in the control group (mean difference: $-3.3,95 \% \mathrm{Cl}-5.7$ to -1.0). The proportion of patients reporting an unacceptable symptom state (IT score over the PASS was $38.9 \%$ in the vaccination group and $46.4 \%$ in the control group (RD $-7.5 \%, 95 \% \mathrm{Cl}-14.4$ to -0.5 , E-value: 1.67) (Table 2).

The effect of vaccination on the severity and impact of long COVID was similar in the subgroup of patients with laboratory confirmed COVID-19. The mean difference in long COVID ST scores was -1.8 , $95 \% \mathrm{Cl}-3.4$ to -0.2 , and the mean difference in long COVID IT scores $-3.8,95 \% \mathrm{Cl}-6.7$ to -0.9

(Supplementary material 7). Similarly, we found no evidence that the effect of vaccination on disease severity varied by time since initial infection (Supplementary material 8 ).

These results were also consistent with a sensitivity analysis where patients who were vaccinated could not be used as controls in periods before their vaccination. The mean difference in long COVID ST scores was $-1.6,95 \% \mathrm{Cl}-3.1$ to -0.2 , and the mean difference in long COVID IT scores was $-3.1,95 \% \mathrm{Cl}-5.8$ to -0.4 (Supplementary material 9). Finally, we investigated whether the delay between baseline and vaccination affected patients' outcomes 120 days after baseline and found no evident interaction (Supplementary material 10).

\section{Serious adverse effects reported in the vaccination group}

All vaccinated patients were asked to report any adverse events that occurred after vaccination and to describe any serious adverse event that may have occurred. In total, 26/455 (5.7\%) patients self-reported a serious adverse effect after vaccination (Table 3$)$, two $(0.4 \%)$ of which led to hospitalisations (one for deep vein thrombosis and the other for meningitis), and two $(0.4 \%)$ led to emergency room visits. Other serious adverse events included relapse of long COVID symptoms $(n=13,2.8 \%)$ and known local and systemic reactions to vaccination (eg, shoulder pain, mild fever, etc.) $(n=5,1 \%)$.

\section{Discussion}

Among patients with long COVID, the first COVID-19 vaccine injection was associated with a reduction in disease severity and impact on patients' lives at 120 days after baseline. In particular, our results show that vaccination doubled the remission rate of long COVID symptoms. Beyond remission, vaccination also significantly increased the proportion of patients reporting an acceptable symptom state by $7.5 \%$. Thus for 13 patients with long COVID who are vaccinated, one will experience a notable decrease in the 
disease's impact on his or her life. Our results also confirm the safety of vaccination for patients with long COVID, with only two $(0.4 \%)$ patients reporting an adverse effect that required hospitalisation.

As of today, millions of patients experience persistent symptoms after a SARS CoV-2 infection and many more may be at risk in the future. To our knowledge, this is the first study of a pharmacological treatment that could reduce the burden of long COVID on care systems.

Our study provides the answer to "one of the four most urgent questions about long COVID" and does so less than 6 months after the first report of remissions of long COVID after vaccination. ${ }^{8} 13$ From a public health perspective, these results are critical in times of vaccine hesitancy, especially among patients who have had a SARS-CoV2 infection and who may fear a relapse or worsening in symptoms. These findings may also game-changing for our understanding of the causes underlying the persistence of symptoms after a SARS-CoV 2 infection: for at least some patients, the disease might be related to at least one of the following mechanisms: 1) presence of a persistent viral reservoir; 2) the presence of virus fragments capable of stimulating the immune system; or 3) development of an autoimmune response induced by the infection. ${ }^{7}$ Future work should explore and describe with precision the symptoms and characteristics of patients who improved after vaccination and could ultimately identify targets for specific therapeutics for long COVID.

From a methodological point of view, our study exemplifies how cohort data can be used to answer comparative effectiveness questions in situations when a randomized trial is not feasible. ${ }^{14}$ Recruitment for such a trial evaluating vaccination among patients with long COVID would be very difficult - if not impossible - in western countries where most of the population has already been vaccinated.

Our study has some limitations. First, despite the use of robust methods and statistical techniques to draw causal inferences from observational data, treatment was not randomly assigned, and potential unmeasured confounders could bias our results. For example, our data did not allow us to take patients' motivation to receive COVID-19 vaccination into account, although it may be related to their perception of their long COVID symptoms and this disease's impact, as measured with the long COVID ST and IT. Second, the administration of questionnaires at regular time intervals did not allow to define follow-up relatively to vaccination. In the time-to-event analysis, study baseline was defined as the vaccination date for patients in the vaccination group, and the vaccination date of their matched patient for those in the control group; the treatment effect reported thus directly related to the efficacy of vaccination at 120 days after vaccination. However, for the analysis of the two patient scores (the long covid ST and IT), baseline was defined as patients' state 120 days before the outcome measurement and up to 60 days before vaccination; the treatment effect reported thus related to the efficacy of vaccination from 60 to 120 days after vaccination. To account for variation in outcome measurement times, we explored how time since vaccination affected the study outcome and found no evidence of an interaction between baseline and time of vaccination on patients' symptoms and impact (Supplementary material 10). 
Third, all patients in our study were infected before May 1, 2021, and thus were not infected with recent variants of concern such as the Delta variant (first confirmed in France in June 2021). The effectiveness of vaccination on persistent symptoms occurring after infection with those variants thus remains unknown.

In conclusion, COVID-19 vaccination reduces the severity and life impact of long COVID among patients with this disease. These results are likely to reduce vaccine hesitancy among patients who have already had COVID-19 and further our knowledge of the mechanisms underlying long COVID.

\section{Conclusion}

This study shows that COVID-19 vaccination reduces the severity and the life impact of long COVID among patients with persistent symptoms.

\section{Declarations}

\section{Data sharing}

All data (including deidentified individual patient data and a data dictionary) from the study are available to academic research teams under the rules of the ComPaRe e-cohort (www.compare.aphp.fr). Studyrelated documents (study protocol, statistical analysis plan, and informed consent form) are available upon request according to the same rules.

\section{Acknowledgments}

The authors thank Elise Diard and Clara Marre from the ComPaRe team and Jo Ann Cahn for editing.

\section{Financial disclosure}

The authors received no specific funding for this work.

\section{Declaration of interests}

The authors declare no competing interests and no financial associations that may be relevant or seen as relevant to the submitted manuscript. The authors have no association with commercial entities that could be viewed as having an interest in the general area of the submitted manuscript.

\section{References}


1. Office for National Statistics. Prevalence of ongoing symptoms following coronavirus (COVID-19) infection in the UK: 2 September 2021. London, UK, 2021.

2. Crook H, Raza S, Nowell J, et al. Long covid-mechanisms, risk factors, and management. BMJ (Clinical research ed) 2021;374:n1648. doi: 10.1136/bmj.n1648 [published Online First: 2021/07/28]

3. Ayoubkhani D, Khunti K, Nafilyan V, et al. Post-covid syndrome in individuals admitted to hospital with covid-19: retrospective cohort study. BMJ (Clinical research ed) 2021;372:n693. doi: 10.1136/bmj.n693 [published Online First: 2021/04/02]

4. Tran V, Porcher R, Pane I, et al. Course of long COVID symptoms over time in the ComPaRe long COVID prospective e-cohort. Nature Communications (submitted) 2021 doi: 10.21203/rs.3.rs753615/v1

5. Davis HE, Assaf GS, McCorkell L, et al. Characterizing long COVID in an international cohort: 7 months of symptoms and their impact. EClinicalMedicine 2021;38:101019. doi: 10.1016/j.eclinm.2021.101019 [published Online First: 2021/07/27]

6. NICE. COVID-19 rapid guideline: managing the long-term effects of COVID-19: NICE, 2020.

7. Massey D, Berrent $D$, Akrami A, et al. Change in Symptoms and Immune Response in People with Post-Acute Sequelae of SARS-Cov-2 Infection (PASC) After SARS-Cov-2 Vaccination. MedRxiv 2021 doi: https://doi.org/10.1101/2021.07.21.21260391

8. LongCovidSOS. The impact of COVID vaccination on symptoms of Long Covid. An international survey of 900 people with lived experience 2021 [Available from: https://3ca26cd7-266e-4609-b25f6f3d1497c4cf.filesusr.com/ugd/8bd4fe_a338597f76bf4279a851a7a4cb0e0a74.pdf accessed 03092021.

9. Arnold D, Milne A, Samms E, et al. Are vaccines safe in patients with Long COVID? A prospective observational study. medRxiv 2021

10. Kuodi. P, Gorelik, Y., Zayyad H, et al. Association between vaccination status and reported incidence of post-acute COVID-19 symptoms in Israel: a cross-sectional study of patients tested between March 2020 and November 2021. medRxiv 2022 doi: https://doi.org/10.1101/2022.01.05.22268800

11. Tran VT, Ravaud P. COllaborative open platform E-cohorts for research acceleration in trials and epidemiology. Journal of clinical epidemiology 2020;124:139-48. doi:

10.1016/j.jclinepi.2020.04.021 [published Online First: 2020/05/08]

12. Tran VT, Riveros C, Clepier B, et al. Development and validation of the long covid symptom and impact tools, a set of patient-reported instruments constructed from patients' lived experience. Clinical infectious diseases: an official publication of the Infectious Diseases Society of America 2021 doi: 10.1093/cid/ciab352 [published Online First: 2021/04/30]

13. Marshall M. The four most urgent questions about long COVID. Nature 2021

14. Hernan MA, Robins JM. Using Big Data to Emulate a Target Trial When a Randomized Trial Is Not Available. American journal of epidemiology 2016;183(8):758-64. doi: 10.1093/aje/kwv254 [published Online First: 2016/03/20] 


\section{Tables}

Table 1: Patients' demographic and clinical characteristics at baseline

$(\mathrm{n}=910)$. Abbreviations: IQR, interquartile range; COPD: chronic obstructive pulmonary disease. 


\begin{tabular}{|c|c|c|c|}
\hline Characteristic & $\begin{array}{c}\text { Total } \\
(\mathrm{n}=910)\end{array}$ & $\begin{array}{l}\text { Vaccinated } \\
\text { patients } \\
(\mathrm{n}=455)\end{array}$ & $\begin{array}{l}\text { Unvaccinated } \\
\text { patients } \\
(\mathrm{n}=455)\end{array}$ \\
\hline \multicolumn{4}{|l|}{ Demographic and clinical data } \\
\hline Age, median (IQR) - years & $\begin{array}{l}47(40- \\
54)\end{array}$ & $47(39-55)$ & $47(40-53)$ \\
\hline Male sex - n (\%) & $\begin{array}{c}177 \\
(19.5)\end{array}$ & $92(20.2)$ & $85(18.7)$ \\
\hline $\begin{array}{l}\text { Educational level }- \text { number }(\%)(\mathrm{n}=909) \\
\text { Middle school or equivalent } \\
\text { High school or equivalent } \\
2 \text { years post-secondary education } \\
\geq 3 \text { years after secondary education } \\
\text { Other }\end{array}$ & $\begin{array}{l}52(5.7) \\
117 \\
(12.9) \\
205 \\
(22.5) \\
515 \\
(56.6) \\
20(2.2) \\
\end{array}$ & $\begin{array}{l}28(6.2) \\
51(11.2) \\
100(22) \\
264(58) \\
11(2.4)\end{array}$ & $\begin{array}{c}24(5.3) \\
66(14.5) \\
105(23.1) \\
251(55.2) \\
9(2)\end{array}$ \\
\hline Presence of at least one comorbidity - n (\%) & $\begin{array}{c}353 \\
(38.8) \\
\end{array}$ & $177(38.9)$ & $175(38.5)$ \\
\hline $\begin{array}{l}\text { Comorbidities - n (\%) } \\
\text { Asthma or COPD } \\
\text { Cardiovascular } \quad \text { diseases } \\
\text { hypertension) } \\
\text { Diabetes } \\
\text { Cancer } \\
\text { Anxiety or depression } \\
\end{array}$ & $\begin{array}{l}67(7.4) \\
36(4) \\
17(1.9) \\
12(1.3) \\
30(3.3) \\
\end{array}$ & $\begin{array}{l}36(7.9) \\
19(4.2) \\
\\
11(2.4) \\
5(1.1) \\
15(3.3) \\
\end{array}$ & $\begin{array}{l}31(6.8) \\
17(3.7) \\
6(1.3) \\
7(1.5) \\
15(3.3) \\
\end{array}$ \\
\hline \multicolumn{4}{|l|}{ COVID-19 data } \\
\hline Confirmed COVID-19 infection $(\mathrm{n}=907)$ & $\begin{array}{c}55 \\
(60.1)\end{array}$ & $271(59.8)$ & $274(60.4)$ \\
\hline $\begin{array}{l}\text { Time from symptom onset to admission, } \\
\text { median (IQR) - months }\end{array}$ & $\begin{array}{l}10.7 \\
(6.4- \\
12.4)\end{array}$ & $\begin{array}{c}11.2 \\
(6.0-12.5)\end{array}$ & $\begin{array}{c}10.6 \\
(8.3-12.2)\end{array}$ \\
\hline Hospitalised for COVID-19 - n (\%) & $81(8.9)$ & $38(8.4)$ & $43(9.5)$ \\
\hline Hospitalised in ICU for COVID-19 - n (\%) & $16(1.8)$ & $7(1.5)$ & $9(2.0)$ \\
\hline $\begin{array}{l}\text { Severity of long COVID at baseline (long } \\
\text { COVID ST) }\end{array}$ & $\begin{array}{c}14(9- \\
21)\end{array}$ & $15(9.5-21)$ & $14(9-21)$ \\
\hline $\begin{array}{l}\text { Impact of long COVID at baseline (long COVID } \\
\text { IT) }(n=907)\end{array}$ & $\begin{array}{l}32(20- \\
43)\end{array}$ & $\begin{array}{l}32.5(20- \\
42)\end{array}$ & $32(21-43)$ \\
\hline \multicolumn{4}{|c|}{\begin{tabular}{|l|l} 
Vaccination data & \\
\end{tabular}} \\
\hline $\begin{array}{l}\text { Vaccine received - } \mathrm{n}(\%) \\
\text { BNT162b2 mRNA } \\
\text { ChAdOx1 } \\
\text { mRNA-1273 } \\
\text { Ad26.COV2.S }\end{array}$ & $\begin{array}{c}359 \\
(39.4) \\
48(5.3) \\
47(5.2) \\
1(0.1) \\
\end{array}$ & $\begin{array}{c}359(78.9) \\
48(10.5) \\
47(10.3) \\
1(0.2)\end{array}$ & - \\
\hline $\begin{array}{l}\text { Number of patients eligible for vaccination } \geq \\
30 \text { days before baseline }\end{array}$ & $\begin{array}{c}116 \\
(12.7)\end{array}$ & $74(16.3)$ & $42(9.2)$ \\
\hline Time from baseline to vaccination in days & $\begin{array}{c}38(24- \\
53)\end{array}$ & $38(24-53)$ & - \\
\hline
\end{tabular}


Table 2: Primary and secondary outcomes at 120 days after baseline $(n=910) .{ }^{1}$ For 275 patients in the control group, data were censored on the date of their vaccination before 120 days [median delay before censoring: 90 days (IQR 72.5 to 105)]. ${ }^{2}$ Outcome data were missing for 37 (8.1\%) and 32 (7.0\%) patients in the vaccination and control groups, respectively. Missing outcome data were handled by multiple imputations with chained equations. ${ }^{3}$ Estimated by the Kaplan-Meier method. Abbreviations: PASS, Patient Acceptable Symptom State, MD, mean difference. RD: risk difference

\begin{tabular}{|c|c|c|c|}
\hline & $\begin{array}{c}\text { Vaccinated } \\
\text { patients } \\
(n=455)\end{array}$ & $\begin{array}{l}\text { Unvaccinated } \\
\text { controls } \\
(n=455)^{1}\end{array}$ & $\begin{array}{l}\text { Difference } \\
(95 \% \mathrm{CI})^{2}\end{array}$ \\
\hline $\begin{array}{l}\text { Long COVID ST score } \\
\text { at } 120 \text { days - Mean (SD) }\end{array}$ & $13(9.4)$ & $14.8(9.8)$ & $\begin{array}{c}\text { MD, }-1.8 \\
(-3.0 \text { to } \\
-0.5)\end{array}$ \\
\hline $\begin{array}{l}\text { Remission of long COVID } \\
\text { by } 120 \text { days }^{3}\end{array}$ & $16.6 \%$ & $7.5 \%$ & $\begin{array}{c}\mathrm{RD}, 9.1 \\
(5.0 \text { to } \\
13.2)\end{array}$ \\
\hline $\begin{array}{l}\text { Long COVID IT score } \\
\text { at } 120 \text { days - Mean (SD) }\end{array}$ & $24.3(16.7)$ & $27.6(16.7)$ & $\begin{array}{c}\mathrm{MD},-3.3 \\
(-5.7 \text { to } \\
-1.0)\end{array}$ \\
\hline $\begin{array}{l}\text { Proportion of patients with a long COVID IT } \\
\text { score }>\text { PASS at } 120 \text { days }\end{array}$ & $38.9 \%$ & $46.4 \%$ & $\begin{array}{c}\mathrm{RD},-7.5 \\
(-14.4 \text { to } \\
0.5)\end{array}$ \\
\hline
\end{tabular}

Table 3: Severe adverse effects self-reported by patients after COVID-19 vaccination in the vaccination group $(n=455)$

\begin{tabular}{|l|c|}
\hline & $\begin{array}{c}\text { Number } \\
(\mathrm{n}=\mathbf{4 5 5})\end{array}$ \\
\hline Deep vein thrombosis, hospitalised & 1 \\
\hline Meningitis, hospitalised & 1 \\
\hline Suspicion of pulmonary embolism, urgent referral to the emergency room & 1 \\
\hline Suspicion of myocarditis, urgent referral to the cardiologist & 1 \\
\hline $\begin{array}{l}\text { Known local and systemic mild and moderate reactions to vaccine (Shoulder } \\
\text { pain, mild or moderate temperature, etc.) }\end{array}$ & 5 \\
\hline Relapse of long COVID symptoms & 13 \\
\hline Heavy legs & 1 \\
\hline Digestive symptoms (e.g., diarrhea) & 2 \\
\hline Fatigue & 1 \\
\hline
\end{tabular}

\section{Figures}




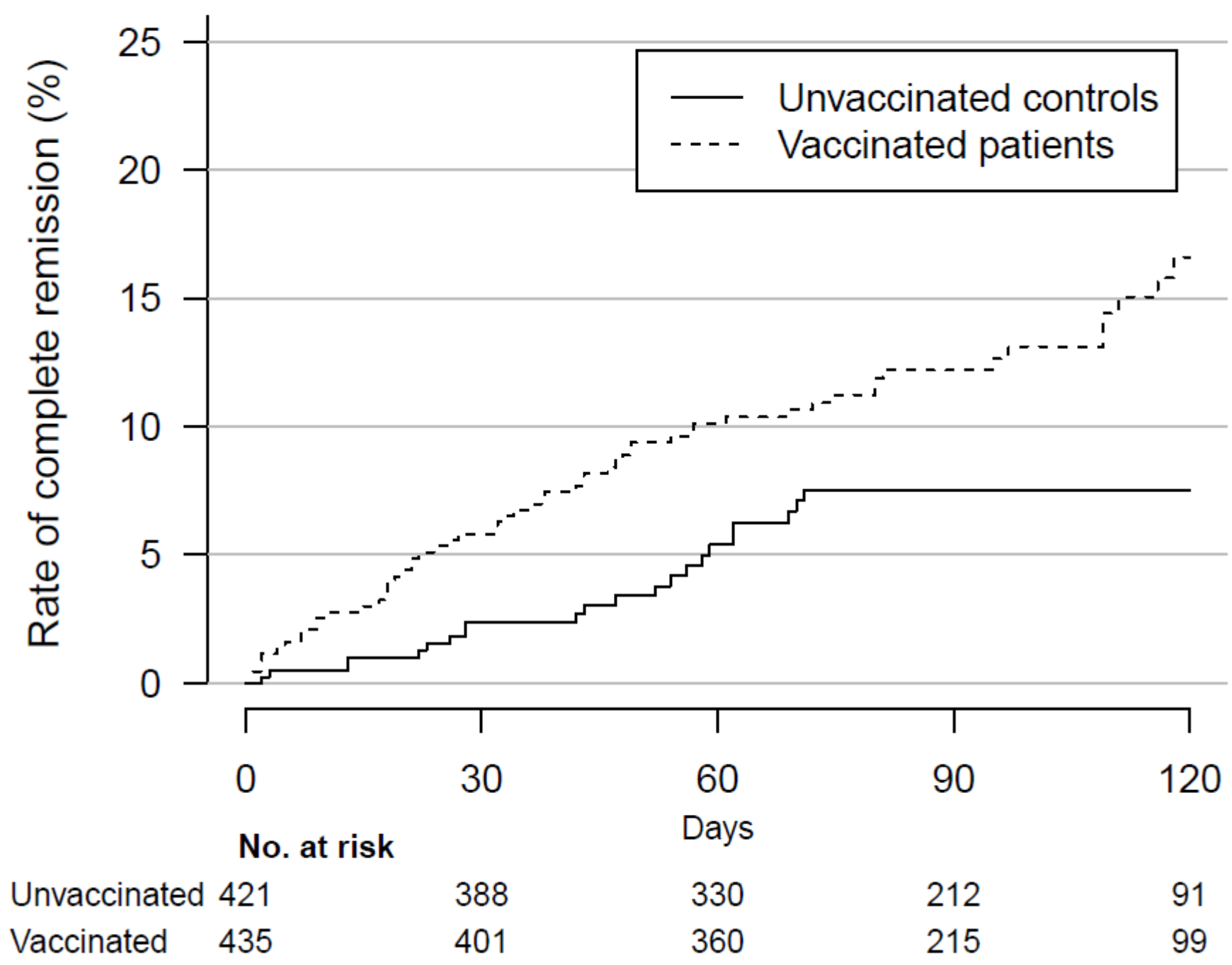

Figure 1

Cumulative event curve for complete remission of long COVID symptoms among vaccinated patients and controls. For 275 patients in the control group, data were censored at the date of their vaccination before 120 days. Artificial censoring was taken into account in survival analyses by inverse probability of censoring weighting methods. In this analysis, time dependant bias was handled by considering the date of vaccination as baseline for vaccinated patients, and the vaccination date of the matched patient as baseline for unvaccinated controls. We thus excluded patients in the vaccination group who achieved symptom remission before vaccination.

\section{Supplementary Files}

This is a list of supplementary files associated with this preprint. Click to download.

- OnlineMethods.docx

- SupplementarymaterialsF.docx 
- nrreportingsummary.pdf

Page $12 / 12$ 